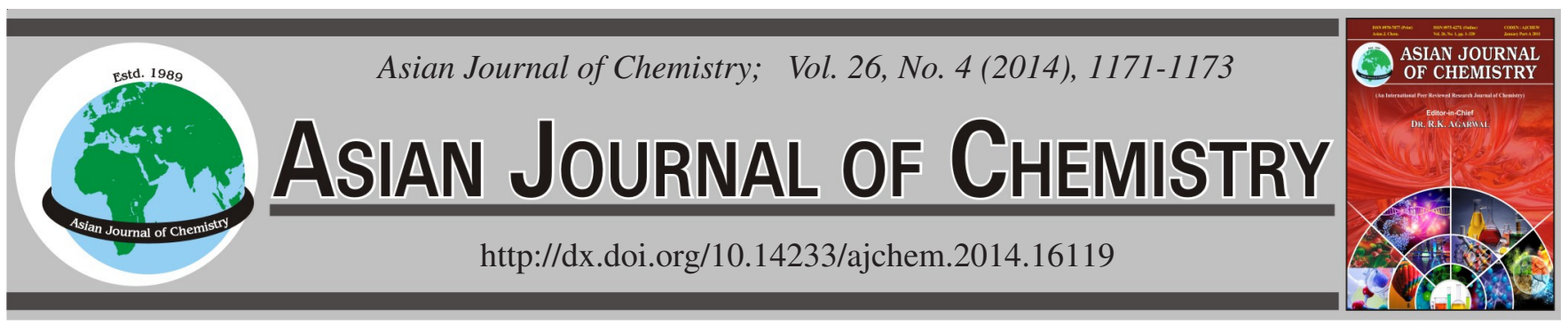

\title{
An Efficient Method for Synthesis of Thiohydantoins with $\alpha$-Amino Esters Under Microwave Irradiation
}

Yanmei Zhao, Zhouyu Wang ${ }^{*}$, Zhenju Jiang ${ }^{*}$, Hualin Feng, Li Liu and Ju Wang

Department of Pharmaceutics Engineering, Xihua University, Chengdu, 610039, P.R. China

*Corresponding authors: Tel: +86 028 7729643; E-mail: zhouyuwang@mail.xhu.edu.cn; zhenjujiang@sina.com

An efficient and simple way for synthesis of thiohydantoins is reported. In the absence of any additional catalysts, a series of thiohydantoins were synthesized with amino esters and isothiocyanates in aqueous medium under microwave irradiation. Excellent isolated yields (up to $98 \%$ ) were obtained under mild conditions.

Keywords: Thiohydantoin, Microwave irradiation, Amino esters, Isothiocyanates.

\section{INTRODUCTION}

Thiohydantoins are the ultimate reaction products of the Edman degradation for peptide sequencing while their derivatives have been found to exist in many nature products and medicines $^{1,2}$. Recent research results showed that thiohydantoins and their derivatives have a wide range of biological activities such as antiviral, antitumor, anticancer, anticonvulsant, antiulcer and antibacterial activities ${ }^{3-9}$. In addition, thiohydantoins are traditionally being considered as useful intermediates in peptide and heterocycles synthesis. For this reason, the synthesis of thiohydantoins is valuable for drug discovery and lots of efficient methods have been reported ${ }^{9-20}$. However, the present methods suffer from drawbacks such as long reaction times, use of corrosive reagents and tedious work-up procedures. Therefore, the development of a simple and efficient method for synthesis of thiohydantoins is still necessary. Herein, we reported a fast and simple way for synthesis of thiohydantoins with $\alpha$-amino esters and isothiocyanates in aqueous medium under the microwave irradiation.

\section{EXPERIMENTAL}

All starting materials were of the commercially available (analytical grade) and used without further purification. All solvents used in the reactions were distilled from appropriate drying agents prior to use. Reactions were monitored by thin layer chromatography using silica gel HSGF254 plates. Flash chromatography was performed using silica gel HG/T235492. Melting poits were measured with SGW X-4 melting point apparatus. ${ }^{1} \mathrm{H}$ and ${ }^{13} \mathrm{C}$ NMR (300 or 600 and 75 or $150 \mathrm{~Hz}$, respectively) spectra were recorded in $\mathrm{CDCl}_{3} .{ }^{1} \mathrm{H} \mathrm{NMR}$ chemical shifts are reported in ppm $(\delta)$ relative to tetramethylsilane
(TMS) with the solvent resonance employed as the internal standard $\left(\mathrm{CDCl}_{3}, \delta 7.26 \mathrm{ppm}\right) .{ }^{13} \mathrm{C}$ NMR chemical shifts are reported in ppm from tetramethylsilane (TMS) with the solvent resonance as the internal standard $\left(\mathrm{CDCl}_{3}, \delta 77.0 \mathrm{ppm}\right)$. IR spectra were recorded on NICONET-380 spectrophotometer using $\mathrm{KBr}$ pellets. ESIMS spectra were recorded on BioTOF Q.

General procedure for the synthesis of thiohydantoins : A mixture of isothiocyanate $(2 \mathrm{mmol})$ and DL-amino ester (2 mmol) were stirred under microwave $(400 \mathrm{w})$ at $400{ }^{\circ} \mathrm{C}$ in $\mathrm{DMF} / \mathrm{H}_{2} \mathrm{O}(3: 1)(8 \mathrm{~mL})$. After $5 \mathrm{~min}$, the solvent was removed under reduced pressure and the residue purified through column chromatography on silica gel (hexane/EtOAc) to give pure product thiohydantoins.

Spectral data of the products: 5-Benzyl-3-phenyl-2thioxoimidazolidin-4-one (3a): White solid; yield: $98 \%$; m.p. 189-190 ${ }^{\circ} \mathrm{C} ;{ }^{1} \mathrm{H}$ NMR $\left(300 \mathrm{MHz}, \mathrm{CDCl}_{3}\right): \delta=3.06-3.14(\mathrm{~m}$, $1 \mathrm{H}), 3.32-3.38(\mathrm{~m}, 1 \mathrm{H}), 4.51-4.54(\mathrm{~m}, 1 \mathrm{H}), 7.07$ (d, $J=9.60$ $\mathrm{Hz}, 2 \mathrm{H}), 7.25-7.51$ (m, 8H), 7.52 (brs, $1 \mathrm{H}) ;{ }^{13} \mathrm{C}$ NMR $(150$ $\left.\mathrm{MHz}, \mathrm{CDCl}_{3}\right): \delta=37.7,60.8,127.9,128.1,129.1,129.3,132.5$, 134.3, 172.5, 183.7; IR (KBr, $\left.v_{\max }, \mathrm{cm}^{-1}\right): 3172,1754,1518$, 1408, 1337, 1269, 1189, 1108; ESI HRMS exact mass calcd. for $\left(\mathrm{C}_{16} \mathrm{H}_{14} \mathrm{~N}_{2} \mathrm{OS}+\mathrm{Na}\right)^{+}$requires $\mathrm{m} / \mathrm{z}$ 305.0719, found $\mathrm{m} / \mathrm{z}$ 305.0719 .

5-Benzyl-3-(4-chlorophenyl)-2-thioxoimidazolidin-4one (3b): White solid; yield: $89 \%$; m.p. $225-227{ }^{\circ} \mathrm{C}$; ${ }^{1} \mathrm{H}$ NMR $\left(300 \mathrm{MHz}, \mathrm{CDCl}_{3}\right): \delta=3.06-3.13(\mathrm{~m}, 1 \mathrm{H}), 3.35-3.38(\mathrm{~m}$, $1 \mathrm{H}), 4.50-4.54(\mathrm{~m}, 1 \mathrm{H}), 7.01(\mathrm{~d}, J=7.23 \mathrm{~Hz}, 2 \mathrm{H}), 7.26$ - $7.43(\mathrm{~m}, 8 \mathrm{H}) ;{ }^{13} \mathrm{C}$ NMR $\left(150 \mathrm{MHz}, \mathrm{CDCl}_{3}\right): \delta=37.7,60.8$, 127.9, 129.1, 129.3, 129.4, 129.5, 130.9, 134.1, 135.3, 172.2, 183.2; IR (KBr, $\left.v_{\max }, \mathrm{cm}^{-1}\right): 3167,1754,1516,1494,1409$, 
1273, 1188, 1092; ESI HRMS exact mass calcd. for $\left(\mathrm{C}_{16} \mathrm{H}_{13} \mathrm{~N}_{2} \mathrm{OSCl}+\mathrm{Na}\right)^{+}$requires $\mathrm{m} / \mathrm{z}$ 339.0329, found $\mathrm{m} / \mathrm{z}$ 339.0341 .

5-Benzyl-3-(4-methoxyphenyl)-2-thioxoimidazolidin4-one (3c): White solid; yield: $90 \%$; m.p. $216-218{ }^{\circ} \mathrm{C} ;{ }^{1} \mathrm{H}$ NMR (300 MHz, $\left.\mathrm{CDCl}_{3}\right): \delta=3.04-3.12(\mathrm{~m}, 1 \mathrm{H}), 3.33-3.38$ $(\mathrm{m}, 1 \mathrm{H}), 3.82(\mathrm{~s}, 3 \mathrm{H}), 4.50-4.54(\mathrm{~m}, 1 \mathrm{H}), 6.93-7.00(\mathrm{~m}, 4 \mathrm{H})$, 7.26-7.28 (m, 3H), 7.34-7.40 (m, 3H); ${ }^{13} \mathrm{C} \mathrm{NMR} \mathrm{(150} \mathrm{MHz,}$ $\left.\mathrm{CDCl}_{3}\right): \delta=37.7,55.4,60.8,125.0,127.8,129.1,129.3,129.4$, 134.3, 172.8, 184.1; IR (KBr, $\left.v_{\max }, \mathrm{cm}^{-1}\right): 3182,1754,1516$, 1408, 1350, 1252, 1172, 1110; ESI HRMS exact mass calcd. for $\left(\mathrm{C}_{17} \mathrm{H}_{16} \mathrm{~N}_{2} \mathrm{O}_{2} \mathrm{~S}+\mathrm{Na}\right)^{+}$requires $\mathrm{m} / \mathrm{z}$ 335.0825, found $\mathrm{m} / \mathrm{z}$ 335.0826.

5-Benzyl-2-thioxo-3-p-tolylimidazolidin-4-one (3d): White solid; yield: $94 \%$; m.p. 214-215 ${ }^{\circ} \mathrm{C}$; ${ }^{1} \mathrm{H}$ NMR (300 $\mathrm{MHz}, \mathrm{CDCl}_{3}$ ): $\delta=2.37$ (s, 3H), 3.07-3.11 (m, 1H), 3.31-3. 33 $(\mathrm{m}, 1 \mathrm{H}), 4.49-4.51(\mathrm{~m}, 1 \mathrm{H}), 6.93(\mathrm{~d}, J=8.16 \mathrm{~Hz}, 2 \mathrm{H}), 7.24-$ 7.37 (m, 7H), 7.62 (brs, $1 \mathrm{H}) ;{ }^{13} \mathrm{C} \mathrm{NMR}\left(150 \mathrm{MHz}, \mathrm{CDCl}_{3}\right): \delta=$ 21.3, 37.6, 60.8, 127.8, 127.9, 129.0, 129.4, 129.9, 134.2, 139.4, 172.7, 183.9; IR (KBr, $\left.v_{\max }, \mathrm{cm}^{-1}\right)$ : 3175, 1753, 1518, 1408, 1347, 1269, 1189, 1112; ESI HRMS exact mass calcd. for $\left(\mathrm{C}_{17} \mathrm{H}_{16} \mathrm{~N}_{2} \mathrm{OS}-\mathrm{H}\right)^{+}$requires $\mathrm{m} / \mathrm{z}$ 295.3901, found $\mathrm{m} / \mathrm{z} 295.0906$.

5-Benzyl-3-methyl-2-thioxoimidazolidin-4-one (3e): White solid; yield: $84 \%$; m.p. $130-132{ }^{\circ} \mathrm{C}$; ${ }^{1} \mathrm{H}$ NMR $(300$ $\left.\mathrm{MHz}, \mathrm{CDCl}_{3}\right): \delta=2.27-2.86(\mathrm{~m}, 1 \mathrm{H}), 3.20(\mathrm{~s}, 3 \mathrm{H}), 3.31-3.37$ (m, 1H), 4.28-4.33 (m, 1H), 7.10 (brs, 1H), 7.20 (d, $J=6.24$ $\mathrm{Hz}, 2 \mathrm{H}), 7.31-7.38(\mathrm{~m}, 3 \mathrm{H}) ;{ }^{13} \mathrm{C} \mathrm{NMR}\left(150 \mathrm{MHz}, \mathrm{CDCl}_{3}\right): \delta=$ 27.5, 37.6, 60.7, 127.4, 129.0, 129.2, 134.9, 173.2, 184.1; IR $\left(\mathrm{KBr}, \mathrm{v}_{\max }, \mathrm{cm}^{-1}\right): 3201,1743,1519,1446,1337,1298,1126$, 1103; ESI MS $(\mathrm{M}+\mathrm{H})^{+} 221.1$.

5-(4-Hydroxybenzyl)-3-methyl-2-thioxoimidazolidin4-one (3f): White solid; yield: $91 \%$; m.p. $178-179{ }^{\circ} \mathrm{C} ;{ }^{1} \mathrm{H}$ NMR (300 MHz, $\left.\mathrm{CDCl}_{3}\right): \delta=2.77-2.81(\mathrm{~m}, 1 \mathrm{H}), 3.20(\mathrm{~s}, 3 \mathrm{H})$, 3.20-3.24 (m,1H), 4.08-4.26 (m, 1H), 5.30 (brs, 1H), 6.80 (d, $J=8.46 \mathrm{~Hz}, 2 \mathrm{H}), 6.91$ (brs, $1 \mathrm{H}), 7.07$ (d, $J=8.40 \mathrm{~Hz}, 2 \mathrm{H})$; ${ }^{13} \mathrm{C}$ NMR $\left(150 \mathrm{MHz}, \mathrm{CDCl}_{3}\right): \delta=27.5,36.8,60.8,126.8$, 130.3, 155.2, 173.2, 184.2; IR (KBr, $\left.v_{\max }, \mathrm{cm}^{-1}\right)$ : 3186, 1736, 1518, 1438, 1338, 1267, 1094; ESI HRMS exact mass calcd. for $\left(\mathrm{C}_{11} \mathrm{H}_{12} \mathrm{~N}_{2} \mathrm{O}_{2} \mathrm{~S}+\mathrm{H}\right)^{+}$requires $\mathrm{m} / \mathrm{z}$ 237.0692, found $\mathrm{m} / \mathrm{z}$ 237.0703 .

3-Methyl-5-phenyl-2-thioxoimidazolidin-4-one (3g): White solid; yield: $88 \%$; m.p. $157-159{ }^{\circ} \mathrm{C}$; ${ }^{1} \mathrm{H}$ NMR $(300$ $\mathrm{MHz}, \mathrm{CDCl}_{3}$ ): $\delta=3.29$ (s, 3H), 5.11 (s, 1H), 7.16 (brs, 1H), 7.31-7.33 (m, 2H), 7.40-7.43 (m, 3H); ${ }^{13} \mathrm{C} \mathrm{NMR} \mathrm{(150} \mathrm{MHz,}$ $\left.\mathrm{CDCl}_{3}\right): \delta=27.8,62.8,126.6,129.3,129.5,133.0,172.3$, 184.7; IR (KBr): 3184, 1758, 1520, 1497, 1273, 1247, 1173, 1108; ESI HRMS exact mass calcd. for $\left(\mathrm{C}_{10} \mathrm{H}_{11} \mathrm{~N}_{2} \mathrm{O}_{2} \mathrm{~S}+\mathrm{H}\right)^{+}$ requires $m / z$, 207.0587, found $m / z, 207.0585$.

3,5-Diphenyl-2-thioxoimidazolidin-4-one (3h): White solid; yield: $96 \%$; m.p. 236-237 ${ }^{\circ} \mathrm{C}$; ${ }^{1} \mathrm{H}$ NMR $(300 \mathrm{MHz}$, $\left.\mathrm{CDCl}_{3}\right): \delta=5.30(\mathrm{~s}, 1 \mathrm{H}), 7.32-7.52(\mathrm{~m}, 10 \mathrm{H}) ;{ }^{13} \mathrm{C}$ NMR $(150$ $\left.\mathrm{MHz}, \mathrm{CDCl}_{3}\right): \delta=63.1,126.6,128.3,128.8,129.2,129.3$, $129.5,129.6,130.9,133.2,171.5,184.2$; IR $\left(\mathrm{KBr}, v_{\max }, \mathrm{cm}^{-1}\right)$ : 3148, 1758, 1520, 1404, 1273, 1183, 1105; ESI HRMS exact mass calcd. for $\left(\mathrm{C}_{15} \mathrm{H}_{12} \mathrm{~N}_{2} \mathrm{OS}+\mathrm{Na}\right)^{+}$requires $\mathrm{m} / z$ 291.0563, found $\mathrm{m} / \mathrm{z}, 291.0565$.

5-Isopropyl-3-phenyl-2-thioxoimidazolidin-4-one (3i): White solid; yield: $91 \%$; m.p. $211-212{ }^{\circ} \mathrm{C}$; ${ }^{1} \mathrm{H}$ NMR $(300$ $\left.\mathrm{MHz} \mathrm{CDCl}_{3}\right): \delta=1.05(\mathrm{~d}, J=6.80 \mathrm{~Hz}, 3 \mathrm{H}), 1.14(\mathrm{~d}, J=6.99$
$\mathrm{Hz}, 3 \mathrm{H}), 2.36-2.40(\mathrm{~m}, 1 \mathrm{H}), 4.11-4.17$ (m, 1H) 7.25-7.30 (m, 2H) 7.46-7.54 (m, 3H), 7.82 (brs, $1 \mathrm{H}) ;{ }^{13} \mathrm{C}$ NMR $(150 \mathrm{MHz}$, $\left.\mathrm{CDCl}_{3}\right): \delta=16.2,18.7,31.6,64.9,128.3,129.2,129.3,132.7$, 172.9, 184.3; IR (KBr, $\left.v_{\max }, \mathrm{cm}^{-1}\right): 3192,1759,1517,1409$, 1349, 1271, 1195, 1108; ESI HRMS exact mass calcd. for $\left(\mathrm{C}_{12} \mathrm{H}_{14} \mathrm{~N}_{2} \mathrm{OS}+\mathrm{Na}\right)^{+}$requires $\mathrm{m} / \mathrm{z}$ 257.0719, found $\mathrm{m} / \mathrm{z}$ 257.0732 .

\section{RESULTS AND DISCUSSION}

The classical method for the synthesis of thiohydantoins is the reaction of isothiocyanates with amino acids. Generally, thiourea was firstly produced and then cyclized to thiohydantoins. For entirely cyclization, acidic or basic conditions are usually required. However, this is not suitable for the synthesis of thiohydantoins with a $\mathrm{pH}$-sensitive group. As part of our program to seek effective, economical and green reactions ${ }^{21-23}$, we wish to synthesize thiohydantoins with amino esters and isothiocyanates in the absence of acid and base. Firstly, we took the reaction of methyl 2-amino-3-phenylpropanoate (1a) and phenyl isothiocyanate (2a) as a model reaction to develop the optimum reaction conditions. When the reaction went through two days in dichloromethane at $40{ }^{\circ} \mathrm{C}$, the yield of $\mathbf{3 a}$ was only $16 \%$. The main product was the thiourea. At the same reaction conditions, we tried other solvents such as toluene, water $\left(\mathrm{H}_{2} \mathrm{O}\right)$ and $\mathrm{N}$, N-dimethylformamide (DMF). There was only trace product in toluene. The yield of 3a were higher in $\mathrm{H}_{2} \mathrm{O}$ and DMF. So we considered use of the mixture of DMF and $\mathrm{H}_{2} \mathrm{O}$ as the solvent. To our surprise, the yield of 3a enhanced to $92 \%$ after two days when $\mathrm{DMF} / \mathrm{H}_{2} \mathrm{O}$ (3:1) was used. Considering the microwave technology has been blossomed into a useful technique in synthetic chemistry due to the fast reaction rate and high yields ${ }^{24-26}$, we used it to increase the efficiency. Much to our surprise, the product yield was enhanced to $98 \%$ in $\mathrm{DMF} / \mathrm{H}_{2} \mathrm{O}(3: 1)$ at $40{ }^{\circ} \mathrm{C}$ after only $5 \mathrm{~min}$ with the power of $400 \mathrm{~W}$. And then, the power of microwave irradiation was studied. When the power was $300 \mathrm{~W}$, the yield of product was decreased to $91 \%$ (entry 7 , Table-1). Further enhancing the power to $600 \mathrm{~W}$ has little effect on the yield (entries 8-9, Table-1). We also investigated the effects of temperature. The results showed that the effects of temperature is the same as the power. Lower temperature disadvantaged the yield and higher temperature has little effect on the yield (entries 10-12, Table-1).

Having established the optimal reaction conditions, we next examined the generality of this microwave-assisted protocol. Various amino esters and isothiocyanates were tested under assistant of microwave irradiation $(400 \mathrm{~W})$ at $40{ }^{\circ} \mathrm{C}$ in the absence of catalysts with $\mathrm{DMF} / \mathrm{H}_{2} \mathrm{O}(3: 1)$ as the solvents. As shown in Scheme-I, the methyl 2-amino-3-phenylpropanoate, methyl 2-amino-3-(4-hydroxy-phenyl)propanoate, methyl 2-amino-2-phenylacetate and methyl 2-amino-3methylbutanoate all cyclized to thiohydantions with phenyl isothiocyanate, 4-chloro-phenyl isothiocyanate,4-methoxyphenyl isothiocyanate, 4-methyl-phenyl isothiocyanate and methyl isothiocyanate to afford the desired thiohydantoins in excellent yields (84-98 \%, Scheme-I).

In summary, we have developed a highly efficient catalystfree method for the synthesis of thiohydantions. With this protocol, a set of thiohydantions can be synthesized with 
TABLE-1

EFFECTS OF REACTION CONDITIONS ON THE SYNTHESIS OF THIOHYDANTOIN ${ }^{a}$

\begin{tabular}{|c|c|c|c|c|c|}
\hline Entry & Solvent & Temp. $\left({ }^{\circ} \mathrm{C}\right)$ & Power $(\mathrm{W})$ & Time & Yield $(\%)^{\mathrm{b}}$ \\
\hline 1 & $\mathrm{CH}_{2} \mathrm{Cl}_{2}$ & 40 & - & $2 \mathrm{~d}$ & 16 \\
\hline 2 & Toluene & 40 & - & $2 d$ & Trace \\
\hline 3 & $\mathrm{H}_{2} \mathrm{O}$ & 40 & - & $2 d$ & 35 \\
\hline 4 & DMF & 40 & - & $2 d$ & 21 \\
\hline 5 & $\mathrm{DMF} / \mathrm{H}_{2} \mathrm{O}(1 / 3)$ & 40 & - & $2 \mathrm{~d}$ & 92 \\
\hline 6 & $\mathrm{DMF} / \mathrm{H}_{2} \mathrm{O}(1 / 3)$ & 40 & 400 & $5 \mathrm{~min}$ & 98 \\
\hline 7 & $\mathrm{DMF} / \mathrm{H}_{2} \mathrm{O}(1 / 3)$ & 40 & 300 & $5 \mathrm{~min}$ & 91 \\
\hline 8 & $\mathrm{DMF} / \mathrm{H}_{2} \mathrm{O}(1 / 3)$ & 40 & 500 & $5 \mathrm{~min}$ & 97 \\
\hline 9 & $\mathrm{DMF} / \mathrm{H}_{2} \mathrm{O}(1 / 3)$ & 40 & 600 & $5 \mathrm{~min}$ & 97 \\
\hline 10 & $\mathrm{DMF} / \mathrm{H}_{2} \mathrm{O}(1 / 3)$ & 20 & 400 & $5 \mathrm{~min}$ & 75 \\
\hline 11 & $\mathrm{DMF} / \mathrm{H}_{2} \mathrm{O}(1 / 3)$ & 60 & 400 & $5 \mathrm{~min}$ & 97 \\
\hline 12 & $\mathrm{DMF} / \mathrm{H}_{2} \mathrm{O}(1 / 3)$ & 80 & 400 & $5 \mathrm{~min}$ & 97 \\
\hline
\end{tabular}<smiles>O=C1C(Cc2ccccc2)NC(=S)N1c1ccccc1</smiles>

3a: $98 \%$ yield<smiles>CC(C)(C)OC(=O)c1ccc(N2C(=O)NC(Cc3ccccc3)C2=S)cc1</smiles><smiles>CN1C(=O)C(c2ccccc2)NC1=S</smiles>

$3 g: 88 \%$ yield<smiles>O=C1C(Cc2ccccc2)NC(=S)N1c1ccc(Cl)cc1</smiles><smiles>CN1C(=O)NC(Cc2ccccc2)C1=S</smiles>

3e: $84 \%$ yield<smiles>CC(C)C1NC(=S)N(c2ccccc2)C1=O</smiles>

3h: $91 \%$ yield<smiles>COc1ccc(N2C(=O)C(Cc3ccccc3)NC2=S)cc1</smiles><smiles>CN1C(=O)NC(Cc2ccc(O)cc2)C1=S</smiles>

3f: $91 \%$ yield<smiles>O=C1NC(c2ccccc2)C(=O)N1c1ccccc1</smiles>

3i: $96 \%$ yield
Scheme-I

excellent yields in absence of any additional catalyst under microwave irradiation.

\section{ACKNOWLEDGEMENTS}

The authors are grateful for the financial supports from the National Natural Science Foundation of China (21102115), the Open Fund of the Key Laboratory of Sichuan Province (Szjj2011-005) and the National Undergraduate Innovation and Entrepreneurship Training Programs of China (201210623009).

\section{REFERENCES}

1. P. Edman, Acta Chem. Scand., 4, 283 (1950).

2. R. Kumar, S. Khan and P. M.S. Chauhan, Curr. Drug Targets, 12, 1689 (2011).
3. A.A. El-Barbary, A.I. Khodair, E.B. Pedersen and C.J. Nielsen, Med. Chem., 37, 73 (1994).

4. B. Mo, J. Li and S. Liang, Anal. Biochem., 249, 207 (1997).

5. E. Osz, L. Somsak, L. Szilagyi, L. Kovacs, T. Docsa, B. Toth and P. Gergely, Bioorg. Med. Chem. Lett., 9, 1385 (1999).

6. R. Kuang, J.B. Epp, S. Ruan, H. Yu, P. Huang, S. He, J. Tu, N.M. Schechter, J. Turbov, C.J. Froelich and W.C. Groutas, J. Am. Chem. Soc., 121, 8128 (1999).

7. Lee, Chen, Liu, JY. CW. S. T.-S. Lee, L.-C. Chen, Y. Liu, J. Wu, Y.-C. Liang and W.-S. Lee, Naunyn Schmiedebergs Arch. Pharmacol., 382, 43 (2010).

8. M.E. Jung, S. Ouk, D. Yoo, C.L. Sawyers, C. Chen, C. Tran and J. Wongvipat, J. Med. Chem., 53, 2779 (2010).

9. M. Ono, S. Hayashi, K. Matsumura, H. Kimura, Y. Okamoto, M. Ihara, R. Takahashi, H. Mori and H. Saji, ACS Chem. Neurosci, 2, 269 (2011).

10. J. Han, H. Dong, Z. Xu, J. Lei and M. Wang, Int. J. Mol. Sci., 14, 12484 (2013).

11. N. Hussain, A. Joshi, C. Sharma and G.L. Talesara, Asian J. Chem., 24, 5917 (2012).

12. D.S. Raghuvanshi and K.N. Singh, Phosphorus Sulfur Silicon Rel. Elem., 185, 2243 (2010).

13. C. Yao, Y. Zhang, G. Zhang, W. Chen, Y. Yu and R.A. Houghten, Synth. Commun., 40, 717 (2010).

14. M. Carboni, J.-M. Gomis, O. Loreau and F. Taran, Synthesis, 417 (2008).

15. S. Cao, L.J. Zhu, C.M. Zhao, X.H. Tang, H.J. Sun, X. Feng and X.H. Qian, Monatsh. Chem., 139, 923 (2008).

16. G.S.M. Sundaram, C. Venkatesh, H. Ila and H. Junjappa, Synlett, 251 (2007).

17. Z.D. Wang, S.O. Sheikh and Y. Zhang, Molecules, 11, 739 (2006).

18. S. Reyes and K. Burgess, J. Org. Chem., 71, 2507 (2006).

19. S. Porwal, R. Kumar, P.R. Maulik and P.M.S. Chauhan, Tetrahedron Lett., 47, 5863 (2006).

20. J.P. Li, C.M. Ma and G.R. Qu, Synth. Commun., 35, 1203 (2005).

21. J. Li, Z. Wang, Z. Jiang, S. Jiang and L. Xiong, Asian J. Chem., 23, 4101 (2011).

22. S. Jiang, Z. Wang, Z. Jiang, J. Li, S. Zhou and L. Pu, Lett. Org. Chem., 9, 24 (2012).

23. S R. S. Caddick and R. Fitzmaurice, Tetrahedron, 65, 3325 (2009).

24. H.M. Hugel, Molecules, 14, 4936 (2009).

25. E.D.. E. Guenin and D. Meziane, Curr. Org. Chem., 15, 3465 (2011).

26. G.K. Gupta, N. Rani and V. Kumar, Mini Rev. Org. Chem., 9, 270 (2012). 